Proceedings of the Edinburgh Mathematical Society (2005) 48, 743-755 (C)

DOI:10.1017/S0013091502000020 Printed in the United Kingdom

\title{
WEIGHTED LIPSCHITZ CONTINUITY AND HARMONIC BLOCH AND BESOV SPACES IN THE REAL UNIT BALL
}

\author{
GUANGBIN REN ${ }^{1}$ AND UWE KÄHLER ${ }^{2}$ \\ ${ }^{1}$ Department of Mathematics, University of Science and Technology of China, \\ Hefei 230026, People's Republic of China (rengb@ustc.edu.cn) \\ ${ }^{2}$ Department of Mathematics, University of Aveiro, \\ 3810-193 Aveiro, Portugal (uwek@mat.ua.pt)
}

(Received 10 January 2002)

\begin{abstract}
The characterization by weighted Lipschitz continuity is given for the Bloch space on the unit ball of $\mathbb{R}^{n}$. Similar results are obtained for little Bloch and Besov spaces.

Keywords: harmonic function theory; Bloch space; Berov space; weighted Lipschitz continuity

2000 Mathematics subject classification: Primary 42B35

Secondary 31B05; 32A50; 32C50
\end{abstract}

\section{Introduction}

Let $\mathbb{B}$ be the unit ball in $\mathbb{R}^{n}$ with $n \geqslant 2$, where $\mathrm{d} \nu$ is the normalized volume measure on $\mathbb{B}$ and $\mathrm{d} \sigma$ is the normalized surface measure on the unit sphere $S=\partial \mathbb{B}$.

In [9], Krantz gave the following extension of a result of Hardy and Littlewood for holomorphic functions on the unit disc (see also $[\mathbf{1 3}]$ and $[\mathbf{8}]$ ).

Theorem 1.1. Let $f$ be a harmonic function on $\mathbb{B}$ and $0<\alpha \leqslant 1$. Then the following are equivalent.

(i) $|\nabla f(x)| \leqslant C\left(1-|x|^{2}\right)^{\alpha-1}$ for any $x \in \mathbb{B}$.

(ii) $|f(x)-f(y)| \leqslant C|x-y|^{\alpha}$ for any $x \in \mathbb{B}$ and $y \in \mathbb{B}$.

In light of this result, it is natural to consider the limit case $\alpha=0$.

The harmonic Bloch space $\mathcal{B}$ is the space of all harmonic functions on $\mathbb{B}$ for which

$$
\sup _{x \in \mathbb{B}}\left(1-|x|^{2}\right)|\nabla f(x)|<\infty,
$$

and the harmonic little Bloch space $\mathcal{B}_{0}$ consists of the functions $f \in \mathcal{B}$ such that

$$
\lim _{|x| \rightarrow 1}\left(1-|x|^{2}\right)|\nabla f(x)|=0 .
$$


The harmonic Besov space $\mathcal{B}_{p}$ is the space of all harmonic functions on $\mathbb{B}$ for which

$$
\int_{\mathbb{B}}\left(1-|x|^{2}\right)^{p}|\nabla f(x)|^{p} \mathrm{~d} \tau(x)<\infty,
$$

where

$$
\mathrm{d} \tau(x)=\left(1-|x|^{2}\right)^{-n} \mathrm{~d} \nu(x)
$$

is the invariant measure on $\mathbb{B}$.

Let $f$ be a continuous function in $\mathbb{B}$. If there exists a constant $C$ such that

$$
\left(1-|x|^{2}\right)^{1 / 2}\left(1-|y|^{2}\right)^{1 / 2}|f(x)-f(y)| \leqslant C|x-y|
$$

for any $x, y \in \mathbb{B}$, then we say that $f$ satisfies a weighted Lipschitz condition.

The main purpose of this paper is to give some characterizations of $\mathcal{B}, \mathcal{B}_{0}$ and $\mathcal{B}_{p}$ by means of a weighted Lipschitz condition. We refer to $[\mathbf{3}, \mathbf{4}, \mathbf{7}, \mathbf{1 0}, \mathbf{1 4}]$ for the corresponding results in the complex unit ball for holomorphic or $\mathcal{M}$-harmonic functions. See $[\mathbf{6}, \mathbf{1 1}$, 15, 17-19] for the various characterizations of the Bloch, little Bloch and Besov spaces in the unit ball of $\mathbb{C}^{n}$. Our main results are the following theorems.

Theorem 1.2. Let $f$ be a harmonic function on $\mathbb{B}$. Then the following are equivalent.

(i) $f \in \mathcal{B}$.

(ii) $f$ satisfies the weighted Lipschitz condition.

Theorem 1.3. Let $f$ be harmonic on $\mathbb{B}$. Then $f \in \mathcal{B}_{0}$ if and only if

$$
\lim _{|x| \rightarrow 1^{-}} \sup \left\{\left(1-|x|^{2}\right)^{1 / 2}\left(1-|y|^{2}\right)^{1 / 2} \frac{|f(x)-f(y)|}{|x-y|}: y \in \mathbb{B}, y \neq x\right\}=0 .
$$

Theorem 1.4. Let $p \in(2(n-1), \infty)$. For any harmonic function $f$ on $\mathbb{B}, f \in \mathcal{B}_{p}$ if and only if

$$
\int_{\mathbb{B}} \int_{\mathbb{B}}\left(1-|x|^{2}\right)^{p / 2}\left(1-|y|^{2}\right)^{p / 2}\left(\frac{|f(x)-f(y)|}{|x-y|}\right)^{p} \mathrm{~d} \tau(x) \mathrm{d} \tau(y)<\infty .
$$

Remark 1.5. Let us remark on the validity of theorem 1.4 that if $p \in(1,2(n-1))$, then the integral condition forces the function to be constant, a fact which is already known in the complex case $(p \in(1,2))$ (see $[\mathbf{1 6}])$.

\section{Preliminaries}

We shall be using the following notation: we will write $x, y \in \mathbb{R}^{n}$ in polar coordinates by $x=|x| x^{\prime}$ and $y=|y| y^{\prime}$.

For any $y, w \in \mathbb{R}^{n}$, the symmetry lemma shows that (see $[\mathbf{2}]$ )

$$
|| y\left|w-y^{\prime}\right|=|| w\left|y-w^{\prime}\right|
$$


The same deduction yields

$$
|| y\left|w-\left(1-|w|^{2}\right) y^{\prime}\right|=|| w\left|y-\left(1-|w|^{2}\right) w^{\prime}\right|
$$

so that

$$
\left.|| y\right|^{2} w-\left(1-|w|^{2}\right) y|=| y|||w| y-\left(1-|w|^{2}\right) w^{\prime} \mid .
$$

For any $a \in \mathbb{B}$, denote by $\varphi_{a}$ the Möbius transformation in $\mathbb{B}$. It is an involutive automorphism of $\mathbb{B}$ such that $\varphi_{a}(0)=\lim _{x \rightarrow 0} \varphi_{a}(x)=a$ and $\varphi_{a}(a)=0$, which is of the form (see $[\mathbf{1}]$ )

$$
\varphi_{a}(x)=\frac{|x-a|^{2} a-\left(1-|a|^{2}\right)(x-a)}{|| x\left|a-x^{\prime}\right|^{2}}, \quad a, x \in \mathbb{B} .
$$

From (2.2) with $w=a$ and $y=x-a$, we have

$$
\left|\varphi_{a}(x)\right|=\frac{|x-a|}{|| a\left|x-a^{\prime}\right|}
$$

whence

$$
1-\left|\varphi_{a}(x)\right|^{2}=\frac{\left(1-|x|^{2}\right)\left(1-|a|^{2}\right)}{|| a\left|x-a^{\prime}\right|^{2}} .
$$

For any $a \in \mathbb{B}$ and $\delta \in(0,1)$, we define

$$
\begin{aligned}
& E(a, \delta)=\left\{x \in \mathbb{B}:\left|\varphi_{a}(x)\right|<\delta\right\}, \\
& B(a, \delta)=\{x \in \mathbb{B}:|x-a|<\delta\} .
\end{aligned}
$$

Clearly, $E(a, \delta)=\varphi_{a}(B(0, \delta))$.

Lemma 2.1. Let $x, w \in \mathbb{B}$ and $y \in E(w, \delta)$. Then

$$
\frac{1-\delta}{1+\delta}|| x\left|w-x^{\prime}\right| \leqslant|| x\left|y-x^{\prime}\right| \leqslant \frac{1+\delta}{1-\delta}|| x\left|w-x^{\prime}\right| .
$$

Proof. From (2.4) and (2.1) we have $\left|\varphi_{y}(w)\right|=\left|\varphi_{w}(y)\right|$, so that $y \in E(w, \delta)$ is equivalent to $w \in E(y, \delta)$. By symmetry, we only have to prove the right-hand inequality. Since ||$x\left|y-x^{\prime}\right| \leqslant|| x|(y-w)|+|| x\left|w-x^{\prime}\right|$, it is enough to show that

$$
|y-w| \leqslant \frac{2 \delta}{1-\delta}|| x\left|w-x^{\prime}\right|
$$

for any $y \in E(w, \delta)$.

Define $\eta=\varphi_{w}(y)$, then $y=\varphi_{w}(\eta)$ and $|\eta|<\delta$. From (2.3), a direct computation yields

$$
\left|\varphi_{w}(\eta)-w\right|=\frac{|\eta|}{|| w\left|\eta-w^{\prime}\right|}\left(1-|w|^{2}\right) .
$$

Therefore, by the simple inequality $1-|w| \leqslant|| x\left|w-x^{\prime}\right|$ we get

$$
|y-w|=\left|\varphi_{w}(\eta)-w\right| \leqslant \frac{\delta}{1-\delta}\left(1-|w|^{2}\right) \leqslant \frac{\delta}{1-\delta} 2|| x\left|w-x^{\prime}\right|
$$

as desired. 
As a direct corollary, we have

$$
1-|x|^{2} \simeq 1-|y|^{2}, \quad x \in E(y, \delta) .
$$

In fact, taking $w=x$ in Lemma 2.1 we get ||$x\left|y-x^{\prime}\right| \simeq 1-|y|^{2}$. The assertion now follows from (2.1).

Lemma 2.2. Let $0<\delta<\lambda$. Then, for any points $x$ and $y$ in $\mathbb{B}$,

$$
\int_{0}^{1} \frac{(1-t)^{\delta-1}}{|t| y\left|x-y^{\prime}\right|^{\lambda}} \mathrm{d} t \leqslant \frac{8^{\lambda}}{\delta(\lambda-\delta)} \frac{1}{|| y\left|x-y^{\prime}\right|^{\lambda-\delta}} .
$$

Proof. Note that for any $t \in[0,1]$ and $x, y \in \mathbb{B}$,

$$
|| y\left|x-y^{\prime}\right| \leqslant 2|t| y\left|x-y^{\prime}\right| .
$$

Indeed, from the triangle inequality we have

$$
\left.\begin{array}{l}
|t| y\left|x-y^{\prime}\right| \geqslant 1-t \\
|t| y\left|x-y^{\prime}\right| \geqslant|| y\left|x-y^{\prime}\right|-(1-t),
\end{array}\right\}
$$

so that summing up yields (2.7).

If ||$y\left|x-y^{\prime}\right| \geqslant 1$, then we have $|t| y\left|x-y^{\prime}\right| \geqslant \frac{1}{2}$ from (2.7). Combining this with the inequality ||$y\left|x-y^{\prime}\right| \leqslant 2$, we get

$$
\int_{0}^{1} \frac{(1-t)^{\delta-1}}{|t| y\left|x-y^{\prime}\right|^{\lambda}} \mathrm{d} t \leqslant \frac{2^{\lambda}}{\delta} \leqslant \frac{2^{2 \lambda-\delta}}{\delta} \frac{1}{|| y\left|x-y^{\prime}\right|^{\lambda-\delta}} .
$$

Now assume that ||$y\left|x-y^{\prime}\right|<1$ and define $r=1-|| y\left|x-y^{\prime}\right|$, then $0<r<1$ and $1-r=|| y\left|x-y^{\prime}\right|$. From (2.7) and (2.8) we have

$$
1-r t=1-t+t|| y\left|x-y^{\prime}\right| \leqslant 3|t| y\left|x-y^{\prime}\right| .
$$

It leads to

$$
\begin{aligned}
\int_{0}^{1} \frac{(1-t)^{\delta-1}}{|t| y\left|x-y^{\prime}\right|^{\lambda}} \mathrm{d} t & \leqslant 3^{\lambda} \int_{0}^{1} \frac{(1-t)^{\delta-1}}{(1-t r)^{\lambda}} \mathrm{d} t \\
& \leqslant 3^{\lambda} \frac{\lambda}{\delta(\lambda-\delta)} \frac{1}{(1-r)^{\lambda-\delta}} \\
& \leqslant C \frac{1}{|| y\left|x-y^{\prime}\right|^{\lambda-\delta}}
\end{aligned}
$$

This completes the proof.

Let $F$ be the hypergeometric function (see $[\mathbf{5}, \mathbf{1 2}]$ )

$$
F(a, b ; c ; s)=\sum_{k=0}^{\infty} \frac{(a)_{k}(b)_{k}}{k !(c)_{k}} s^{k}
$$

for $a, b, c \in \mathbb{R}$ and $c$ neither zero nor a negative integer, where the Pochhammer symbol $(a)_{0}=1$ and $(a)_{k}=a(a+1) \cdots(a+k-1), k \in \mathbb{N}$. We need some known properties of 
hypergeometric functions:

(i) Bateman's integral formula (see [12])

$$
F(a, b ; c+\mu ; s)=\frac{\Gamma(c+\mu)}{\Gamma(c) \Gamma(\mu)} \int_{0}^{1} t^{c-1}(1-t)^{\mu-1} F(a, b ; c ; t s) \mathrm{d} t
$$

with $c, \mu>0$ and $s \in(-1,1)$; and

(ii) for any integer $m[\mathbf{1 2}$, p. 69],

$$
\left.\begin{array}{c}
F(-m, b ; c ; 1)=\frac{(c-b)_{m}}{(c)_{m}}, \\
F(-m, a+m ; c ; 1)=\frac{(-1)^{m}(1+a-c)_{m}}{(c)_{m}} .
\end{array}\right\}
$$

The following identity furnishes the hypergeometric function with an integral representation.

Lemma 2.3. Let $t>1, \lambda \in \mathbb{R}$ and $r \in(-1,1)$, then

$$
\int_{-1}^{1} \frac{\left(1-u^{2}\right)^{(t-3) / 2}}{\left(1-2 r u+r^{2}\right)^{\lambda}} \mathrm{d} u=\frac{\Gamma\left(\frac{1}{2}(t-1)\right) \Gamma\left(\frac{1}{2}\right)}{\Gamma\left(\frac{1}{2} t\right)} F\left(\lambda, \lambda+1-\frac{1}{2} t ; \frac{1}{2} t ; r^{2}\right) .
$$

Proof. Let $C_{m}^{\lambda}(u)$ be the Gegenbauer polynomials. They can be defined by the generating function

$$
\left(1-2 r u+r^{2}\right)^{-\lambda}=\sum_{m=0}^{\infty} C_{m}^{\lambda}(u) r^{m}
$$

where

$$
\left.\begin{array}{rl}
C_{2 m}^{\lambda}(u) & =(-1)^{m} \frac{(\lambda)_{m}}{m !} F\left(-m, m+\lambda ; \frac{1}{2} ; u^{2}\right), \\
C_{2 m+1}^{\lambda}(u) & =(-1)^{m} \frac{(\lambda)_{m}}{m !} 2 u F\left(-m, m+\lambda+1 ; \frac{3}{2} ; u^{2}\right) .
\end{array}\right\}
$$

To calculate the integral in (2.11), we apply (2.12) and (2.13). Then we deduce that it is only needed to evaluate the integral

$$
\int_{-1}^{1}\left(1-u^{2}\right)^{(t-3) / 2} F\left(-m, m+\lambda ; \frac{1}{2} ; u^{2}\right) \mathrm{d} u,
$$

or, rather, an integral over the interval $(0,1)$ by the simple change of variables $t=u^{2}$. For this integral, we first use Bateman's integral formula (2.9) with $s=1$ and apply (2.10), so that it can be represented by Pochhammer symbols. The calculation of the integral in (2.11) then leads to a series which by definition is the desired hypergeometric function. 
Lemma 2.4. Let $\alpha>-1$ and $\beta \in \mathbb{R}$. Then, for any $x \in \mathbb{B}$,

$$
\int_{\mathbb{B}} \frac{\left(1-|y|^{2}\right)^{\alpha}}{|| x\left|y-x^{\prime}\right|^{n+\alpha+\beta}} \mathrm{d} y \approx \begin{cases}\left(1-|x|^{2}\right)^{-\beta}, & \beta>0, \\ \log \frac{1}{1-|x|^{2}}, & \beta=0, \\ 1, & \beta<0 .\end{cases}
$$

The notation $a(x) \approx b(x)$ means that the ratio $a(x) / b(x)$ has a positive finite limit as $|x| \rightarrow 1$.

Proof. Denote the above integral by $J_{\alpha, \beta}(x)$. From Stirling's formula we need only show that

$$
J_{\alpha, \beta}(x)=\frac{\Gamma\left(\frac{1}{2} n+1\right) \Gamma(\alpha+1)}{\Gamma\left(\alpha+\frac{1}{2} n+1\right)} F\left(\frac{1}{2}(n+\alpha+\beta), \frac{1}{2}(2+\alpha+\beta) ; \alpha+\frac{1}{2} n+1 ;|x|^{2}\right) .
$$

For any continuous function $f$ of one variable and any $\eta \in \partial \mathbb{B}$, we have the formula (see [2, p. 216])

$$
\int_{\partial \mathbb{B}} f(\langle\zeta, \eta\rangle) \mathrm{d} \sigma(\zeta)=\frac{\Gamma\left(\frac{1}{2} n\right)}{\Gamma\left(\frac{1}{2}(n-1)\right) \Gamma\left(\frac{1}{2}\right)} \int_{-1}^{1}\left(1-u^{2}\right)^{(n-3) / 2} f(u) \mathrm{d} u,
$$

where $\langle\cdot, \cdot\rangle$ denotes the inner product in $\mathbb{R}^{n}$. Taking

$$
f(u)=\left(1-2 r u+r^{2}\right)^{-(n+\alpha+\beta) / 2}
$$

for fixed $r \in(0,1)$ and combining with Lemma 2.3 we have

$$
\begin{aligned}
\int_{\partial \mathbb{B}}\left(1-2 r\langle\zeta, \eta\rangle+r^{2}\right)^{-(n+\alpha+\beta) / 2} \mathrm{~d} \sigma(\zeta) & \\
= & \frac{\Gamma\left(\frac{1}{2} n\right)}{\Gamma\left(\frac{1}{2}(n-1)\right) \Gamma\left(\frac{1}{2}\right)} \int_{-1}^{1} \frac{\left(1-u^{2}\right)^{(n-3) / 2}}{\left(1-2 r u+r^{2}\right)^{(n+\alpha+\beta) / 2}} \mathrm{~d} u \\
= & F\left(\frac{1}{2}(n+\alpha+\beta), \frac{1}{2}(2+\alpha+\beta) ; \frac{1}{2} n ; r^{2}\right) .
\end{aligned}
$$

Consequently, from the polar coordinates formula we get

$$
\begin{aligned}
J_{\alpha, \beta}(x) & =n \int_{0}^{1} r^{n-1}\left(1-r^{2}\right)^{\alpha} \int_{S}\left(1-2 r|x|\left\langle x^{\prime}, \eta\right\rangle+r^{2}|x|^{2}\right)^{-(n+\alpha+\beta) / 2} \mathrm{~d} \sigma(\zeta) \\
& =C \int_{0}^{1} r^{n-1}\left(1-r^{2}\right)^{\alpha} F\left(\frac{1}{2}(n+\alpha+\beta), \frac{1}{2}(2+\alpha+\beta) ; \frac{1}{2} n ; r^{2}|x|^{2}\right) \mathrm{d} r .
\end{aligned}
$$

The assertion now follows from Bateman's integral formula (2.9).

\section{Bloch space}

In this section we give the proof of Theorems 1.2 and 1.3. Theorem 1.2 can be rewritten in the following form. 
Theorem 3.1. For any harmonic function $f$ on $\mathbb{B}, f \in \mathcal{B}$ if and only if

$$
\sup \left\{\left(1-|x|^{2}\right)^{1 / 2}\left(1-|y|^{2}\right)^{1 / 2} \frac{|f(x)-f(y)|}{|x-y|}: x, y \in \mathbb{B}, x \neq y\right\}<\infty .
$$

Proof. Assume that $f \in \mathcal{B}$. For any $x, y \in \mathbb{B}$, we have

$$
\begin{aligned}
f(x)-f(y) & =\int_{0}^{1} \frac{\mathrm{d} f}{\mathrm{~d} t}(t x+(1-t) y) \mathrm{d} t \\
& =\sum_{k=1}^{n}\left(x_{k}-y_{k}\right) \int_{0}^{1} \frac{\partial f}{\partial x_{k}}(t x+(1-t) y) \mathrm{d} t .
\end{aligned}
$$

By the Cauchy-Schwarz inequality and the simple inequality

$$
\left|\frac{\partial f}{\partial x_{k}}\right| \leqslant|\nabla f|
$$

we have

$$
\begin{aligned}
|f(x)-f(y)| & \leqslant \sqrt{\sum_{k=1}^{n}\left|x_{k}-y_{k}\right|^{2}} \sqrt{\sum_{k=1}^{n}\left(\int_{0}^{1}\left|\frac{\partial f}{\partial x_{k}}(t x+(1-t) y)\right| \mathrm{d} t\right)^{2}} \\
& \leqslant|x-y| \sqrt{n} \int_{0}^{1}|(\nabla f)(t x+(1-t) y)| \mathrm{d} t .
\end{aligned}
$$

Since $\left(1-|t x+(1-t) y|^{2}\right)|(\nabla f)(t x+(1-t) y)| \leqslant\|f\|_{\mathcal{B}}$, it follows that

$$
\frac{|f(x)-f(y)|}{|x-y|} \leqslant \sqrt{n} C\|f\|_{\mathcal{B}} \int_{0}^{1} \frac{1}{1-|t x+(1-t) y|^{2}} \mathrm{~d} t .
$$

Now, by the triangle inequality $|t x+(1-t) y| \leqslant t|x|+(1-t)|y|$, we have

$$
1-|t x+(1-t) y| \geqslant(1-t)(1-|x|)
$$

and

$$
1-|t x+(1-t) y| \geqslant t(1-|y|)
$$

so that

$$
\begin{aligned}
\int_{0}^{1} \frac{1}{1-|t x+(1-t) y|} \mathrm{d} t & \leqslant \int_{0}^{1} \frac{1}{\{(1-t)(|1-| y \mid)\}^{1 / 2}} \frac{1}{\{t(|1-| x \mid)\}^{1 / 2}} \mathrm{~d} t \\
& =\pi \frac{1}{(1-|x|)^{1 / 2}(1-|y|)^{1 / 2}} .
\end{aligned}
$$

Thus

$$
\left(1-|x|^{2}\right)^{1 / 2}\left(1-|y|^{2}\right)^{1 / 2} \frac{|f(x)-f(y)|}{|z-w|} \leqslant 2 \pi \sqrt{n} C\|f\|_{\mathcal{B}} .
$$

This proves the necessity. 
Conversely, suppose that $f$ is harmonic and that (3.1) is satisfied. We will show that $f \in \mathcal{B}$.

Fix $\delta \in(0,1)$. Since $f$ is harmonic, combining the result in [11, p. 504] with (2.7) we get

$$
\left(1-|x|^{2}\right)|\nabla f(x)| \leqslant C \int_{E(x, \delta)}|f(y)| \mathrm{d} \tau(y) .
$$

Fixing $x \in \mathbb{B}$ and replacing $f$ by $f-f(x)$, we have

$$
\left(1-|x|^{2}\right)|\nabla f(x)| \leqslant C \int_{E(x, \delta)}|f(y)-f(x)| \mathrm{d} \tau(y) .
$$

Therefore,

$$
\left(1-|x|^{2}\right)|\nabla f(x)| \leqslant C \sup \{|f(y)-f(x)|: w \in E(x, \delta)\} .
$$

Note that for any $y \in E(x, \delta)$ we have $\left|\varphi_{x}(y)\right| \leqslant \delta$, and thus

$$
\frac{\sqrt{1-\left|\varphi_{x}(y)\right|^{2}}}{\left|\varphi_{x}(y)\right|} \geqslant C
$$

It follows from Lemma 2.1 that

$$
\frac{\left(1-|x|^{2}\right)^{1 / 2}\left(1-|y|^{2}\right)^{1 / 2}}{|y-x|} \geqslant C, \quad y \in E(x, \delta) .
$$

Consequently,

$$
\left(1-|x|^{2}\right)|\nabla f(x)| \leqslant C \sup \left\{\frac{\left(1-|x|^{2}\right)^{1 / 2}\left(1-|y|^{2}\right)^{1 / 2}}{|y-x|}|f(y)-f(x)|: y \in E(x, \delta)\right\},
$$

in which the right-hand side can be controlled by the condition in (3.1). This implies $f \in \mathcal{B}$ and completes the proof of Theorem 3.1.

Theorem 3.2. For any harmonic function $f$ on $\mathbb{B}, f \in \mathcal{B}_{0}$ if and only if

$$
\lim _{|x| \rightarrow 1^{-}} \sup \left\{\left(1-|x|^{2}\right)^{1 / 2}\left(1-|y|^{2}\right)^{1 / 2} \frac{|f(x)-f(y)|}{|x-y|}: y \in \mathbb{B}, y \neq x\right\}=0 .
$$

Proof. Assume that $f \in \mathcal{B}_{0}$. Let $f_{t}(x)=f(t x), t \in(0,1)$. By (3.1), we have

$$
\left(1-|x|^{2}\right)^{1 / 2}\left(1-|y|^{2}\right)^{1 / 2} \frac{\left|\left(f-f_{t}\right)(x)-\left(f-f_{t}\right)(y)\right|}{|x-y|} \leqslant C\left\|f-f_{t}\right\|_{\mathcal{B}}
$$

and

$$
\begin{aligned}
\left(1-|x|^{2}\right)^{1 / 2}(1 & \left.-|y|^{2}\right)^{1 / 2} \frac{\left|f_{t}(x)-f_{t}(y)\right|}{|x-y|} \\
& =t \frac{\left(1-|x|^{2}\right)^{1 / 2}\left(1-|y|^{2}\right)^{1 / 2}}{\left(1-|t x|^{2}\right)^{1 / 2}\left(1-|t y|^{2}\right)^{1 / 2}}\left(1-|t x|^{2}\right)^{1 / 2}\left(1-|t y|^{2}\right)^{1 / 2} \frac{|f(t x)-f(t y)|}{|t x-t y|} \\
& \leqslant C \frac{t}{\left(1-t^{2}\right)^{1 / 2}}\left(1-|x|^{2}\right)^{1 / 2}\|f\|_{\mathcal{B} .}
\end{aligned}
$$


By the triangle inequality, we obtain

$$
\begin{aligned}
\sup \left\{\left(1-|x|^{2}\right)^{1 / 2}\left(1-|y|^{2}\right)^{1 / 2} \frac{|f(x)-f(y)|}{|x-y|}\right. & : y \in \mathbb{B}, y \neq x\} \\
& \leqslant C \frac{t}{1-t^{2}}\left(1-|x|^{2}\right)^{1 / 2}\|f\|_{\mathcal{B}}+\left\|f-f_{t}\right\|_{\mathcal{B}} .
\end{aligned}
$$

In the above inequality, $t \rightarrow 0$ as $|x| \rightarrow 1^{-}$the first term on the right-hand side converges to 0 , and afterwards if $t \rightarrow 1^{-}$, then the second term on the right-hand side also converges to 0 , which is similar to the case $n=2$ which can be found in $[\mathbf{2 0}]$.

Now suppose that $f$ is harmonic and (3.6) is satisfied. We will show that $f \in \mathcal{B}_{0}$.

We remind the reader that (the invariant gradient) $|\tilde{\nabla} f(x)|$ is defined as

$$
|\tilde{\nabla} f(x)|=\left(1-|x|^{2}\right)|\nabla f(x)| .
$$

From (3.3) and (3.4) we have

$$
|\tilde{\nabla} f(x)| \leqslant C(n, r) \int_{E(x, r)}\left(1-|x|^{2}\right)^{1 / 2}\left(1-|y|^{2}\right)^{1 / 2} \frac{|f(x)-f(y)|}{|x-y|} \mathrm{d} \tau(y) .
$$

By the assumption (3.6), for any given $\epsilon>0$ there exists $\delta \in(0,1)$ such that

$$
\sup \left\{\left(1-|x|^{2}\right)^{1 / 2}\left(1-|y|^{2}\right)^{1 / 2} \frac{|f(x)-f(y)|}{|x-y|}: y \in \mathbb{B}, y \neq x\right\}<\epsilon
$$

whenever $|x|>\delta$. Since

$$
\int_{E(x, r)} \mathrm{d} \tau=\tau(E(a, \delta))=\tau(B(0, \delta))=n \int_{0}^{\delta} t^{n-1}\left(1-t^{2}\right)^{-n} \mathrm{~d} t,
$$

we have

$$
|\tilde{\nabla} f(x)|<C \epsilon
$$

for any $|z|>\delta$, which means that $|\tilde{\nabla} f(x)| \rightarrow 0$ as $|z| \rightarrow 1^{-}$. This completes the proof.

\section{Besov spaces}

In this section, we give a higher-dimensional form of the Holland-Walsh characterization for Besov spaces. When $p \rightarrow \infty$, it also reveals the weighted Lipschitz characterization of the Bloch space.

Theorem 4.1. Let $p \in(2(n-1), \infty)$ and let $f$ be harmonic on $\mathbb{B}$. Then $f \in \mathcal{B}_{p}$ if and only if

$$
\int_{\mathbb{B}} \int_{\mathbb{B}}\left(1-|x|^{2}\right)^{p / 2}\left(1-|y|^{2}\right)^{p / 2}\left(\frac{|f(x)-f(y)|}{|x-y|}\right)^{p} \mathrm{~d} \tau(x) \mathrm{d} \tau(y)<\infty .
$$

To prove Theorem 4.1, we need the following lemma.

For this lemma we remind the reader that for $x \in \mathbb{B}$ we have

$$
\tilde{\nabla} f(x)=\nabla\left(f \circ \varphi_{x}\right)(0) .
$$


Lemma 4.2. Let $p \geqslant 1$ and let $f$ be harmonic on $\mathbb{B}$. Then

$$
\int_{\mathbb{B}}\left(\int_{0}^{1} \frac{|\tilde{\nabla} f(t a)|}{1-t|a|} \mathrm{d} t\right)^{p} \mathrm{~d} \nu_{\alpha}(a) \leqslant C \int_{\mathbb{B}}|\tilde{\nabla} f(a)|^{p} \mathrm{~d} \nu_{\alpha}(a),
$$

where $\mathrm{d} \nu_{\alpha}(a)=\left(1-|a|^{2}\right)^{\alpha} \mathrm{d} \nu(a), \alpha>-1$.

For the proof we remind the reader that the Hardy-Littlewood integral means that $M_{p}(r,|f|)$ is defined as

$$
M_{p}(r,|f|)=\int_{\partial \mathbb{B}}|f(r \zeta)|^{p} \mathrm{~d} \sigma(\zeta) .
$$

Proof of Lemma 4.2. Fix $\epsilon \in(0,1)$. Let $t \in[0,1], a \in \mathbb{B}$. If at least one of $t$ and $|a|$ is less than $\epsilon$, then $|t a|=t|a|<\epsilon$, so

$$
\frac{1}{1-t|a|} \leqslant \frac{1}{1-\epsilon}
$$

and the left-hand side of (4.2) can be controlled by

$$
\int_{\mathbb{B} \backslash \in \mathbb{B}}\left(\int_{\epsilon}^{1} \frac{|\tilde{\nabla} f(t a)|}{1-t|a|} \mathrm{d} t\right)^{p} \mathrm{~d} \nu_{\alpha}(a)+C \sup _{x \in \in \mathbb{B}}|\tilde{\nabla} f(x)|^{p} .
$$

Denote the first summand above by $I$. From the polar coordinates integral formula and Minkowski's inequality we have

$$
\begin{aligned}
I & =n \int_{\epsilon}^{1} \int_{\partial \mathbb{B}}\left(\int_{\epsilon}^{1} \frac{|\tilde{\nabla} f(t s \zeta)|}{1-t s} \mathrm{~d} t\right)^{p} \mathrm{~d} \sigma(\zeta) s^{n-1}\left(1-s^{2}\right)^{\alpha} \mathrm{d} s \\
& \leqslant C \int_{\epsilon}^{1}\left(\int_{\epsilon}^{1} \frac{M_{p}(t s,|\tilde{\nabla} f|)}{1-t s} \mathrm{~d} t\right)^{p} s^{n-1}\left(1-s^{2}\right)^{\alpha} \mathrm{d} s \\
& \leqslant C \int_{\epsilon}^{1}\left(\int_{\epsilon^{2}}^{s} h(\rho) \mathrm{d} \rho\right)^{p}\left(1-s^{2}\right)^{\alpha} \mathrm{d} s,
\end{aligned}
$$

where

$$
h(\rho)=\frac{\rho^{(n-1) / p} M_{p}(\rho,|\tilde{\nabla} f|)}{1-\rho} .
$$

Applying Hardy's inequality

$$
\int_{0}^{1}\left(\int_{0}^{s} h(\rho) \mathrm{d} \rho\right)^{p}(1-s)^{\alpha} \mathrm{d} s \leqslant C \int_{0}^{1} h^{p}(t)(1-t)^{\alpha+1} \mathrm{~d} t,
$$

which follows from Hölder's inequality and Fubini's theorem, we have

$$
\begin{aligned}
I & \leqslant C \int_{0}^{1}\left(\int_{0}^{s} h(\rho) \mathrm{d} \rho\right)^{p}(1-s)^{\alpha} \mathrm{d} s \\
& \leqslant C \int_{0}^{1} t^{n-1}(1-t)^{\alpha} M_{p}^{p}(t,|\tilde{\nabla} f|) \mathrm{d} t \\
& =C \int_{\mathbb{B}}|\tilde{\nabla} f(a)|^{p} \mathrm{~d} \nu_{\alpha}(a) .
\end{aligned}
$$


It remains to show that

$$
\sup _{x \in \mathbb{B}}|\tilde{\nabla} f(x)|^{p} \leqslant C \int_{\mathbb{B}}|\tilde{\nabla} f(a)|^{p} \mathrm{~d} \nu_{\alpha}(a) .
$$

The starting point to prove this is the following inequality:

$$
|g(x)| \leqslant C \int_{E(x, \delta)}|g(w)| \mathrm{d} \tau(w)
$$

which holds for any harmonic function $g$. Since each partial derivative of a harmonic function remains harmonic, we have

$$
|\nabla f(x)| \leqslant C \int_{E(x, \delta)}|\nabla f(w)| \mathrm{d} \tau(w)
$$

Recall that $|\tilde{\nabla} f(x)|=\left(1-|x|^{2}\right)|\nabla f(x)|$ and $1-|w| \simeq 1-|x|$ for any $w \in E(x, \delta)$ and $a \in \mathbb{B}$, hence we have

$$
|\tilde{\nabla} f(x)| \leqslant C \int_{E(x, \delta)}|\tilde{\nabla} f(w)| \mathrm{d} \tau(w)
$$

Because $1-|w| \simeq 1-|x| \simeq 1$ for any $w \in E(x, \delta)$ and $x \in \epsilon \mathbb{B}$, applying Hölder's inequality we get

$$
\begin{aligned}
|\tilde{\nabla} f(x)|^{p} & \leqslant C \int_{E(x, \delta)}|\tilde{\nabla} f(w)|^{p} \mathrm{~d} \tau(w) \\
& \leqslant C \int_{E(x, \delta)}|\tilde{\nabla} f(w)|^{p} \mathrm{~d} \nu_{\alpha}(w) .
\end{aligned}
$$

The assertion (4.3) now follows. This finishes the proof of Lemma 4.2.

Proof of Theorem 4.1. Assume that $f \in C^{\infty}(\mathbb{B})$. For any $a \in \mathbb{B}$, we have

$$
\frac{|f(a)-f(0)|}{|a|}=\left|\int_{0}^{1} \nabla f(t a) \frac{a}{|a|} \mathrm{d} t\right| \leqslant \int_{0}^{1} \frac{|\tilde{\nabla} f(t a)|}{1-t|a|} \mathrm{d} t .
$$

It follows from Lemma 4.2 that

$$
\begin{aligned}
\int_{\mathbb{B}} \frac{|f(a)-f(0)|^{p}}{|a|^{p}} \mathrm{~d} \nu_{\alpha}(a) & \leqslant C \int_{\mathbb{B}}\left(\int_{0}^{1} \frac{|\tilde{\nabla} f(a)|}{1-t|a|} \mathrm{d} t\right)^{p} \mathrm{~d} \nu_{\alpha}(a) \\
& \leqslant C \int_{\mathbb{B}}|\tilde{\nabla} f(a)|^{p} \mathrm{~d} \nu_{\alpha}(a) .
\end{aligned}
$$


Replacing $f$ with $f \circ \varphi_{x}$, integrating with respect to $\mathrm{d} \tau(x)$, taking $y=\varphi_{x}(a)$, and setting $\alpha=p / 2-n$, we have

$$
\begin{aligned}
& \int_{\mathbb{B}} \int_{\mathbb{B}} \frac{|f(y)-f(x)|^{p}}{\left|\varphi_{x}(y)\right|^{p}}\left(1-\left|\varphi_{x}(y)\right|^{2}\right)^{p / 2} \mathrm{~d} \tau(x) \mathrm{d} \tau(y) \\
& \leqslant C \int_{\mathbb{B}} \int_{\mathbb{B}}|\tilde{\nabla} f(y)|^{p}\left(1-\left|\varphi_{x}(y)\right|^{2}\right)^{p / 2} \mathrm{~d} \tau(x) \mathrm{d} \tau(y) \\
& \leqslant C \int_{\mathbb{B}}|\tilde{\nabla} f(y)|^{p} \mathrm{~d} \tau(y) \int_{\mathbb{B}}\left(1-\left|\varphi_{x}(y)\right|^{2}\right)^{p / 2} \mathrm{~d} \tau(x) \\
& \leqslant C \int_{\mathbb{B}}|\tilde{\nabla} f(y)|^{p} \mathrm{~d} \tau(y) .
\end{aligned}
$$

In the last step, we used the estimate

$$
\int_{\mathbb{B}}\left(1-\left|\varphi_{x}(y)\right|^{2}\right)^{p / 2} \mathrm{~d} \tau(x) \leqslant C
$$

for $p>2(n-1)$, which follows from $(2.5)$ and Lemma 2.4. Since

$$
\frac{\left(1-\left|\varphi_{x}(y)\right|^{2}\right)^{p / 2}}{\left|\varphi_{x}(y)\right|^{p}}=\frac{\left(1-|x|^{2}\right)^{p / 2}\left(1-|y|^{2}\right)^{p / 2}}{|x-y|^{p}}
$$

we get (4.1).

Conversely, suppose that $f$ is harmonic and that it satisfies (4.1), we will show that $f \in B_{p}$. For any fixed $\delta \in(0,1)$,

$$
|\tilde{\nabla} f(x)| \leqslant C \int_{E(x, \delta)}|f(x)-f(y)| \mathrm{d} \tau(y) .
$$

Then, by applying Hölder's inequality and (3.4) we obtain

$$
\begin{aligned}
|\tilde{\nabla} f(x)|^{p} & \leqslant C \int_{E(x, \delta)}|f(x)-f(y)|^{p} \mathrm{~d} \tau(y) \\
& \leqslant \int_{E(x, \delta)}|f(x)-f(y)|^{p} \frac{\left(1-|x|^{2}\right)^{p / 2}\left(1-|y|^{2}\right)^{p / 2}}{|x-y|^{p}} \mathrm{~d} \tau(y) .
\end{aligned}
$$

Thus, (4.1) implies $f \in B_{p}$. This completes the proof.

Acknowledgements. G.R. was supported by the NNSF of China (under grant nos 10001030, 19871081) and by a postdoctoral fellowship from the University of Aveiro, UI\&D 'Mathematics and Applications'.

\section{References}

1. L. V. Ahlfors, Möbius transformations in several dimensions, Ordway Professorship Lectures in Mathematics (University of Minnesota, Minneapolis, MN, 1981).

2. S. Axler, P. Bourdon and W. Ramey, Harmonic function theory, Graduate Texts in Mathematics, vol. 137 (Springer, 1992). 
3. J. BuRBEA AND S. Y. LI, Weighted Hadamard products of holomorphic functions in the ball, Pac. J. Math. 168 (1995), 235-270.

4. J. S. Choe, H. O. Kim And Y. Y. PARK, A Bergman-Carleson measure characterization of Bloch functions in the unit ball of $\mathbb{C}^{n}$, Bull. Kor. Math. Soc. 29 (1992), 285-293.

5. A. ERdÉLYi et al., Higher transcendental functions, vol. I (McGraw-Hill, New York, 1953).

6. K. T. Hahn And E. H. Youssfi, Möbius invariant Besov p-spaces and Hankel operators in the Bergman space on the ball in $\mathbb{C}^{n}$, Complex Variables 17 (1991), 89-104.

7. F. Holland AND D. WAlsh, Criteria for membership of Bloch space and its subspace, BMOA, Math. Ann. 273 (1986), 317-335.

8. T. Kiso, Y. Mizuta and T. Shimomura, A theorem of Hardy-Littlewood for harmonic functions satisfying Hölder's condition, J. Math. Soc. Jpn 47 (1995), 121-130.

9. S. G. KRAntz, Lipschitz spaces, smoothness of functions, and approximation theory, Expo. Math. 3 (1983), 193-260.

10. M. NowAK, Bloch space and Möbius invariant Besov spaces on the unit ball on $\mathbb{C}^{n}$, Complex Variables 44 (2001), 1-12.

11. C. H. OUYAng, W. S. YAng AND R. H. ZhaO, Characterizations of Bergman spaces and Bloch space in the unit ball of $\mathbb{C}^{n}$, Trans. Am. Math. Soc. 374 (1995), 4301-4312.

12. E. D. Rainville, Special functions (Chelsea, New York, 1971).

13. W. Rudin, Holomorphic Lipschitz functions in balls, Comment. Math. Helv. 53 (1978), 143-147.

14. W. Rudin, Function theory in the unit ball of $\mathbb{C}^{n}$ (Springer, 1980).

15. K. Stroethoff, Besov type characterization for the Bloch space, Bull. Aust. Math. Soc. 39 (1989), 405-420.

16. K. Stroethoff, The Bloch space and Besov spaces of analytic functions, Bull. Aust. Math. Soc. 54 (1996), 211-219.

17. R. M. Timoney, Bloch functions in several complex variables, I, Bull. Lond. Math. Soc. 12 (1980), 241-267.

18. R. M. Timoney, Bloch functions in several complex variables, II, J. Reine Angew. Math. 319 (1980), 1-22.

19. R. YonedA, A characterization of the harmonic Bloch space and the harmonic Besov spaces by an oscillation, Proc. Edinb. Math. Soc. 45 (2002), 229-239.

20. K. ZHU, Operator theory in function spaces (Marcel Dekker, New York, 1990). 